\title{
COMPORTAMENTO DE CULTIVARES DE COQUEIROS ANÃO E HÍBRIDOS NO DISTRITO FEDERAL ${ }^{1}$
}

\author{
VÍCTOR HUGO VARGAS RAMOS², ALBERTO CARLOS DE QUEIROZ PINTO³, WILSON MENEZES ARAGÃO4, \\ ANTONIO CARLOS GOMES ${ }^{5}$, NILTON TADEU VILELA JUNQUEIRA ${ }^{6}$, EDSON LOBATO ${ }^{7}$, \\ MARIA ALICE SANTOS OLIVEIRA ${ }^{8}$
}

\begin{abstract}
RESUMO - O cultivo do coqueiro na região Centro-Oeste pode tornar-se importante opção devido à crescente demanda por água de coco e à necessidade de os produtores diversificarem as atividades e melhorarem a renda. A demanda é totalmente atendida até hoje pela oferta de frutos de outros estados, tendo sido comercializadas 1.700 t via CEASA-DF, em 1994. Apesar da importância econômica, a produtividade nacional é baixa pelo uso de genótipos não selecionados e pouca informação a respeito das práticas culturais.Com o objetivo de avaliar cultivares e híbridos de coqueiro quanto ao crescimento vegetativo, a Embrapa Cerrados-DF, em parceria com a Embrapa Tabuleiros Costeiros, desenvolve o experimento em delineamento de blocos ao acaso, com quatro e dois tratamentos, respectivamente, de cultivares e de híbridos, com três repetições. Os tratamentos foram as cultivares: Anão-vermelho-da-malásia, Anão-verde-de-jiqui, Anão-vermelho-de-camarões e Anão-vermelho-de-gramame, e os híbridos: Anão-amarelode-gramame x Gigante-do-oeste-africano e Anão-vermelho-de-gramame x Gigante-do-brasil-da-praia-do-forte. As plantas foram avaliadas quanto ao crescimento, à circunferência do coleto, ao número e ao comprimento de folhas. O objetivo desta pesquisa foi avaliar cultivares e híbridos de coqueiro quanto ao crescimento vegetativo, visando a indicar os materiais com melhor aptição para o cultivo no DF. Das cultivares, o 'Anão-verde-de-jiqui' vem-se destacando no crescimento e na circunferência do coleto, e, entre os híbridos, não houve diferença .
\end{abstract}

Termos para indexação: Cocos nucifera, mudas, avaliação, sistema de produção, cerrados.

\section{BEHAVIOR OF DWARF CULTIVARS AND HYBRIDS OF COCONUT IN BRASÍLIA , FEDERAL DISTRICT}

ABSTRACT-The coconut tree has become an important crop in Brazilian Central Region. Profitability, high demand for coconut water and the grower needs for supplementary source of income are the main factors responsible for the increasing interest for cultivation of coconut tree. A total of 1.700 $t$ of coconut was commercialized at CEASA-DF in 1994 mainly imported from other regions. Despite the importance of this crop, the average of national yield is very low due to the use of unselected genotypes and undefined production system.The Embrapa Cerrados Research Center has started a partnership work with Embrapa Tabuleiros Costeiros to evaluate the behavior of dwarf cultivars and hybrids regarding vegetative growth in Brasília, Federal District. This study was established in a randomized block design with four and two treatments of dwarf and hybrid genotypes and three replications for each study. The dwarf varieties were the following: Red-Dwarf-from-Malasia (RDM), Green-Dwarf-from-Jiquí (GDJ), Red-Dwarf-fromCameroon (RDC), Red-Dwarf-from-Gramame (RDG); and the hybrids: Yellow-Dwarf-from-Gramame x Giant-from-Western-Africa (YDG x GWA), RedDwarf-from-Gramame x Brazilian-giant-from-Praia-do-Forte (RDG x BGPF). The following growth parameters were evaluated: trunk (stipe) circumference, number and length of leaves. The 'Green Dwarf of Jiqui' has shown the highet growth of the trunk circumference among the dwarf genotypes, but no difference has been found among hybrids.

Index terms: Cocos nucifera, nursery, selection, production system, Brazilian Cerrados.

O cultivo do coqueiro (Cocos nucifera $\mathrm{L}$ ) vem-se tornando cada vez mais atrativo pela crescente demanda por água de coco e pela necessidade de os produtores buscarem fontes alternativas de renda. A demanda é totalmente atendida pela oferta de frutos provenientes de outros estados, tendo sido comercializadas $1.700 \mathrm{t}$ via CEASA-DF, em 1994 (Lima, 1995).

Em condições de seca, o crescimento do coqueiro é retardado, e a produtividade, reduzida (Mahindapala \& Pinto, 1991).

$\mathrm{Na}$ região Centro-Oeste, baixas temperaturas durante determinados períodos do ano e elevadas taxas de evapotranspiração, associadas à irregularidade na distribuição das chuvas, provocam deficits hídricos, fator limitante da produtividade, com abortamento de flores. Esse deficit constitui o principal fator limitante no desenvolvimento do coqueiro que, por ter crescimento e produção contínuos, exige condições de clima muito próximas do ideal durante todo o ano (Passos,1998b.).

O objetivo desta pesquisa foi avaliar cultivares e híbridos de coqueiro quanto ao crescimento vegetativo, visando a indicar os materiais com melhor aptição para o cultivo no DF.

O trabalho foi desenvolvido na Embrapa Cerrados, em Planaltina, Distrito Federal (latitude $17^{\circ} 35^{\prime} 03^{\prime}$, , longitude $47^{\circ} 42$ ' 30 ' e altitude de $1.100 \mathrm{~m}$ ). O clima da região tem duas estações: a chuvosa e quente com precipitação de 1.400 a $1.800 \mathrm{~mm} /$ ano e temperatura de 25 a $30{ }^{\circ} \mathrm{C}$ de setembro a abril, e a seca, com umidade relativa em torno de $40 \%$ e temperatura entre 20 e $23{ }^{\circ} \mathrm{C}$ de maio a agosto. Os experimentos foram instalados em 8-2-2000, numa área de 1,5 ha. Foram usadas as cultivares anãs: Anão-verde-de-jiqui (AVeJ), Anão-vermelho-de-gramame (AVG), Anão-vermelho-da-malásia (AVM) e Anão-vermelho-de-camarões (AVC) e os híbridos: Anão-vermelho-de-gramame x Gigante-do-brasil-da-praiado-forte (AVG x GBRPF) e Anão-amarelo-de-gramame x Gigante-do-oesteafricano (AAG x GOA).

Para as cultivares anãs, o delineamento experimental utilizado foi o de blocos ao acaso, com quatro tratamentos (cultivares) e três repetições. A unidade experimental para as cultivares anãs (AVeJ) foi constituída por 12 plantas e 5 plantas para as cultivares (AVG, AVM e AVC). O espaçamento utilizado foi de 7,5 x 7,5 x 7,5 m, em triângulo eqüilátero, em covas de $60 \times 60 \times 60 \mathrm{~cm}$. Para os híbridos, foi em blocos ao acaso, com dois tratamentos (híbridos) e três repetições. A unidade experimental dos híbridos foi constituída por 12 plantas. O espaçamento utilizado foi de $8,5 \times 8,5 \times 8,5 \mathrm{~m}$, em triângulo eqüilátero, com as mesmas dimensões de covas dos anões. Os dois experimentos estão sendo conduzidos em condições de sequeiro, com a irrigação suplementar por microaspersão, implantada em nov/2003, em dois turnos de rega de 8

\footnotetext{
${ }^{1}$ (Trabalho 007/2004). Recebido: 02/01/2004. Aceito para publicação: 04/08/2004.

${ }^{2}$ Eng. Agrôn., Ph.D., Embrapa Cerrados, Caixa Postal 08223, CEP 73301-970, Planaltina-DF. (vhugo@cpac.embrapa.br).

${ }^{3}$ Eng. Agrôn., Ph.D., Embrapa Cerrados, alcapi@cpac.embrapa.br.

${ }^{4}$ Eng. Agrôn., D.Sc., Embrapa Tabuleiros Costeiros, Caixa Postal 44, CEP 45025-040, Aracajú-SE (aragaowm@ cpatc.embrapa.br).

${ }^{5}$ Mat. Bioest., D.Sc., Embrapa Cerrados, acarlos@cpac.embrapa.br.

${ }^{6}$ Eng. Agrôn., Ph.D., Embrapa Cerrados, junqueir@ cpac.embrapa.br.

${ }^{7}$ Eng. Agrôn., M.Sc., Embrapa Cerrados, lobato@cpac.embrapa.br.

${ }^{8}$ Eng. Agrôn., M.Sc., Embrapa Cerrados, alice@ @pac.embrapa.br.
} 
TABELA 1 - Características químicas da área experimental -Embrapa Cerrados -Novembro 1999

\begin{tabular}{|c|c|c|c|c|c|c|c|}
\hline Áreas & Profundidade $(\mathrm{cm})$ & $\mathrm{P}\left(\mathrm{mg} / \mathrm{dm}^{3}\right)$ & $\mathrm{pH}$ em água & $\mathrm{K}\left(\mathrm{mg} / \mathrm{dm}^{3}\right)$ & $\mathrm{Ca}+\mathrm{Mg}\left(\mathrm{cmol}_{\mathrm{c}} / \mathrm{dm}^{3}\right)$ & $\mathrm{H}+\mathrm{Al}\left(\mathrm{cmol}_{\mathrm{c}} / \mathrm{dm}^{3}\right)$ & $\mathrm{Al}\left(\mathrm{cmol}_{\mathrm{c}} / \mathrm{dm}^{3}\right)$ \\
\hline \multirow[t]{2}{*}{1} & $0-30$ & 2,5 & 5,4 & 63 & 2,5 & 8,4 & 0,7 \\
\hline & $30-60$ & 0,5 & 4,8 & 29 & 0,9 & 8,4 & 1,1 \\
\hline \multirow[t]{2}{*}{2} & $0-30$ & 6,3 & 5,5 & 94 & 4,1 & 9,2 & 0,2 \\
\hline & $30-60$ & 1,0 & 4,7 & 34 & 1,1 & 10,3 & 1,2 \\
\hline \multirow[t]{2}{*}{3} & $0-30$ & 2,6 & 5,6 & 95 & 3,2 & 6,2 & 0,2 \\
\hline & $30-60$ & 0,3 & 4,9 & 28 & 1,1 & 6,5 & 0,7 \\
\hline
\end{tabular}

Fonte: Tabela elaborada pelo Laboratório de solos da Embrapa Cerrados, 1999.

horas por dia e por semana, com uma vazão de 30 L.h ${ }^{-1}$. O solo utilizado foi o Latossolo Vermelho-Amarelo, argilo-arenoso.

A área experimental apresentava variação em suas características químicas (Tabela 1). Assim sendo, foram aplicadas doses diferenciadas de calcário dolomítico de 3,2 t/ha (Área 1), 2,7 t/ha (Área 2) e 1,7 t/ha (Área 3) para se atingir 50\% de saturação por bases e doses de $\mathrm{P}$ sob a forma de Superfosfato simples de $900 \mathrm{~kg} / \mathrm{ha}$, nas 3 áreas a lanço e incorporadas. A aplicação de gesso foi de 3,0 t/há, nas 3 áreas, e a adubação na cova de plantio foi de $5 \mathrm{~kg}$ de casca picada de coco no fundo da cova, $10 \mathrm{~kg}$ de esterco de curral, FTE -BR 12, $50 \mathrm{gr}, 800 \mathrm{~g}$ de superfosfato simples, inseticida clorpirifós 20 gr e misturado com a terra da superfície para encher a cova.

As avaliações foram realizadas com 1,$5 ; 16,5 ; 19,0 ; 23,0$ e 26,0 meses após o plantio no campo: (a) Altura da planta (AP) : da superfície do solo até o ápice da folha central, com auxílio de uma régua graduada em centímetros; (b) Circunferência do coleto do estipe (CC): efetuada a 5 centímetros do solo, com auxílio de uma fita métrica; (c) Número de folhas vivas (NFV): número de folhas verdes e adultas com mais de $50 \%$ da folha aberta; (d) Número de folhas mortas (NFM): número de folhas adultas com mais de $90 \%$ dos folíolos secos;..(e) Número de folhas emitidas (NFE): número de folhas com abertura superior a 50\%, incluindo as vivas e as mortas, desde a última avaliação; (f) Comprimento da folha três (CF3): determinado com auxílio de uma régua graduada em centímetros, contada a partir da primeira folha aberta do centro da planta, em sentido anti-horário (Aragão, 1998:// www.cpatsa.embrapa.br/livrorg/ coco.doc);.(g) Comprimento do pecíolo na folha três (CPF3): comprimento em centímetros entre a inserção da folha três no estipe até o $1^{\circ}$ folíolo

Os dados obtidos foram submetidos à análise de variância e comparados pelo teste de Tukey, a 5\% de probabilidade. Utilizou-se a análise de variância repetida no tempo, na análise de regressão, para os parâmetros altura da planta e circunferência no coleto do estipe.

Verificou-se que houve diferença significativa $(\mathrm{p}=0,05)$ na altura da cultivar Anão-verde-de-jiqui (Tabela 2) em relação à cultivar Anãovermelho-de-camarões, não diferindo do Anão-vermelho-de-gramame e da Malásia. Quanto à circunferência no coleto do estipe, observa-se que houve diferença significativa da cultivar Anão-verde-de-jiqui, em relação às outras três cultivares. Isso pode sugerir, até o momento, o melhor desempenho dessa cultivar nas condições edafoclimáticas do DF. Não houve interação significativa entre as épocas e as cultivares para as variáveis altura da planta e circunferência no coleto do estipe. O crescimento em altura do coqueiro-anão nas épocas estudadas $(1,5$; 16,$5 ; 19,0 ; 23,0$ e 26,0 meses) após o plantio no campo, independentemente da cultivar, apresentou resposta quadrática: $\mathrm{y}=0,72-0,02 \mathrm{X}+0,0029 \mathrm{X}^{2}$ , $\mathrm{R}^{2}=0,99(\mathrm{p}=0,01)$, indicando que o crescimento com o tempo $(\mathrm{X}=$ número de meses) é igual para as cultivares anãs. $\mathrm{O}$ mesmo acontece com a circunferência do coleto, cuja resposta foi: $\mathrm{y}=10,8-0,417 \mathrm{X}+$ $0,07094 \mathrm{X}^{2}, \mathrm{R}^{2}=0,98(\mathrm{p}=0,01)$, indicando aumento da circunferência no tempo igual para as cultivares. As cultivares anãs tiveram crescimento médio em altura de $5,77 \mathrm{~cm} /$ mês, enquanto o aumento da circunferência do coleto foi de $1,45 \mathrm{~cm} / \mathrm{mês}$.

Em relação ao desempenho dos dois híbridos (Tabela 3), a CC do híbrido AAG x GOA com 26,0 meses de idade é 42,8\% menor que o relatado por Marcílio et al.( 2001), com o mesmo híbrido e idade da planta, enquanto a CC do híbrido AVG x GBRPF é $36,8 \%$ menor do que o encontrado por Marcílio et al.( 2001), com o mesmo híbrido. Esse resultado
TABELA 2 - Avaliação da altura (m) e circunferência do coleto $(\mathrm{cm})$ da planta das cultivares de coqueiro-anão nas épocas $(1,5 ; 16,5$; 19,0; 23,0 e 26,0 meses), aos 26 meses após o plantio no campo. Embrapa Cerrados. Planaltina - DF. Agosto - 2002.

\begin{tabular}{lcc}
\hline Cultivares & $\begin{array}{c}\text { Altura da } \\
\text { planta }(\mathrm{m})\end{array}$ & $\begin{array}{c}\text { Circunferência } \\
\text { do coleto }(\mathrm{cm})\end{array}$ \\
\hline Anão-verde-de-jiqui & $1,71 \mathrm{a}$ & $36,16 \mathrm{a}$ \\
Anão-vermelho-de-gramame & $1,51 \mathrm{a} \mathrm{b}$ & $30,11 \mathrm{~b}$ \\
Anão-vermelho-da-malásia & $1,41 \mathrm{a} \mathrm{b}$ & $28,27 \mathrm{~b}$ \\
Anão-vermelho-de-camarões & $1,24 \mathrm{~b}$ & $25,96 \mathrm{~b}$ \\
\hline CV (\%) (Cultivar) & 7,58 & 6,13 \\
CV(\%) (Cultivar x Época) & 7,16 & 11,48 \\
DMS (Tukey a 5\%) & 0,31 & 5,23 \\
Teste F & $9,2 * *$ & $16,7 * *$ \\
\hline
\end{tabular}

Médias seguidas de letras diferentes na coluna diferem estatisticamente, pelo teste de Tukey, a 5\% de probabilidade. ** significativo a $1 \%$.

de menor $\mathrm{CC}$ indica menor desenvolvimento vegetativo, conforme Mahindapala \& Pinto (1991).

Quanto aos híbridos, não houve interação significativa entre épocas e híbridos para as variáveis altura e circunferência do coleto. $\mathrm{O}$ crescimento em altura dos coqueiros híbridos, nas épocas estudadas, independentemente do híbrido, apresentou resposta quadrática: $\mathrm{y}=1,19-0,0134 \mathrm{X}+0,0039 \mathrm{X}^{2}$ , $\mathrm{R}^{2}=0,99,(\mathrm{p}=0,01)$, indicando que o crescimento no tempo ( $X=$ número de meses) foi igual para os híbridos. O mesmo acontece com a circunferência do coleto: $\mathrm{y}=15,02-1,0528 \mathrm{X}+0,1351 \mathrm{X}^{2}, \mathrm{R}^{2}=0,97,(\mathrm{p}=0,01)$, indicando aumento da circunferência no tempo igual para os híbridos. Os híbridos tiveram crescimento médio em altura de $8,88 \mathrm{~cm} / \mathrm{mês}$, enquanto o aumento da circunferência do coleto foi de $2,49 \mathrm{~cm} / \mathrm{mês}$.

As médias obtidas no vigésimo sexto mês, para o número de folhas vivas (Tabela 4), foram significativamente maiores para o Anãoverde-de-jiqui $(8,69)$ em relação ao Anão-vermelho-de-camarões $(7,43)$, enquanto o número de folhas emitidas também foi significativamente maior para o Anão-verde-de-jiqui $(13,69)$ em relação ao Anão-vermelhoda-malásia $(11,54)$ e ao Anão-vermelho-de-camarões $(10,88)$. Esses valores são menores que os obtidos em condições ambientais consideradas favoráveis, onde um coqueiro-anão pode emitir até 18 folhas/ano (Passos, 1998 a.). Ainda se compararmos o NFE $(13,69)$ pela cultivar AVeJ, o NFV $(8,69)$ e NFM $(5,00)$ às 11 folhas vivas e 1 morta, verificado por Jucá et al. (2002), com essa mesma cultivar irrigada, percebe-se a necessidade da instalação de irrigação sob o risco de não explorar o potencial genético das cultivares estudadas.

TABELA 3 - Avaliação da altura da planta $(m)$ e circunferência do coleto (cm) da planta dos híbridos de coqueiro, nas épocas $(1,5$; 16,$5 ; 19,0 ; 23,0$ e 26,0 meses) aos 26 meses após o plantio no campo. Embrapa - Cerrados. Planaltina - DF. Agosto - 2002.

\begin{tabular}{lcc}
\hline Híbridos & $\begin{array}{c}\text { Altura da } \\
\text { planta }(\mathrm{m})\end{array}$ & $\begin{array}{c}\text { Circunferência do } \\
\text { coleto }(\mathrm{cm})\end{array}$ \\
\hline AAG x GOA & $2,49 \mathrm{a}$ & $49,56 \mathrm{a}$ \\
AVG x GBRPF & $2,35 \mathrm{a}$ & $45,16 \mathrm{a}$ \\
\hline CV (\%) (Híbrido) & 5,12 & 6,41 \\
CV(\%) (Híbrido x Época) & 4,35 & 7,82 \\
DMS (Tukey a 5\%) & 0,43 & 10,67 \\
Teste F & $1,92 \mathrm{~ns}$ & $3,14 \mathrm{~ns}$ \\
\hline
\end{tabular}

Médias seguidas de letras diferentes na coluna diferem estatisticamente, pelo teste de Tukey, a 5\% de probabilidade. ns - não significativo 
TABELA 4 - Avaliação do NFV, NFM, NFE, CF3 e CPF3, de quatro cultivares de coqueiro-anão, aos 26 meses após o plantio no campo. Embrapa Cerrados. Planaltina - DF. Agosto - 2002.

\begin{tabular}{lccccc}
\hline Cultivares & NFV $\left(\mathrm{n}^{\mathrm{o}}\right)$ & NFM $\left(\mathrm{n}^{\circ}\right)$ & NFE $\left(\mathrm{n}^{\circ}\right)$ & CF3 $(\mathrm{cm})$ & CPF3 $(\mathrm{cm}))$ \\
\hline AVeJ & $8,69 \mathrm{a}$ & 5,00 & $13,69 \mathrm{a}$ & $158,8 \mathrm{a}$ & $20,1 \mathrm{c}$ \\
AVG & $8,00 \mathrm{a} \mathrm{b}$ & 4,40 & $12,40 \mathrm{ab}$ & $154,8 \mathrm{a}$ & $26,0 \mathrm{~b}$ \\
AVM & $7,45 \mathrm{a} \mathrm{b}$ & 4,09 & $11,54 \mathrm{~b}$ & $152,7 \mathrm{a}$ & $27,6 \mathrm{a} \mathrm{b}$ \\
AVC & $7,43 \mathrm{~b}$ & 3,45 & $10,88 \mathrm{~b}$ & $145,0 \mathrm{a}$ & $31,2 \mathrm{a}$ \\
\hline CV (\%) & 11,91 & & 9,64 & 14,0 & 15,5 \\
DMS (Tukey a 5\%) & 1,25 & & 1,57 & 28,09 & 4,89 \\
Teste F & $7,5 * *$ & $19,4 * *$ & $1,26 \mathrm{~ns}$ & $30,24 * *$ \\
\hline
\end{tabular}

Médias seguidas de letras diferentes na coluna diferem estatisticamente, pelo teste de Tukey, a 5\% de probabilidade.** significativo a $1 \%$. ns - não significativo. NFM - não foi feita análise estatística

Houve diferença no CPF3 da cultivar AVC $(31,2 \mathrm{~cm})$ em relação à cultivar AVeJ (20,1 cm) e AVG (26,0) (Tabela 4). OCF3, com 158,8 cm e o CPF3 com 20,1 cm para o AVeJ aos 26 meses, neste trabalho, quando comparado ao AVeJ irrigado estudado por Jucá et al. (2002), com valores de $193 \mathrm{~cm}$ e $27 \mathrm{~cm}$, respectivamente. Esse desempenho menor dessa cultivar em Brasília, das características em estudo, indicam falta de irrigação.

As médias obtidas no vigésimo sexto mês, para o número de folhas vivas, variaram de 8,05 para o híbrido AVG x GBRPF a 9,05 para o híbrido AAG x GOA, não havendo diferença estatística entre eles (Tabela 5).Neste caso, o desenvolvimento vegetativo foi menor, em relação ao dos híbridos estudados por Marcílio et al. (2001), que apresentaram valores entre 12,5 para o híbrido AVG x GBRPF e 12,1 para o híbrido AAG x GOA, aos 26 meses depois do plantio, e isso também pode ser atribuído à falta de irrigação.

A taxa de produção de folhas emitidas avaliada no período de 26,0 meses pelo híbrido AAG x GOA foi 14,38 folhas, e não diferiu do híbrido AVG x GBRPF com 12,54 folhas, neste mesmo período.

TABELA 5 - Avaliação do NFV, NFM, NFE, CF3 e CPF3, dos híbridos de coqueiro, aos 26 meses após o plantio no campo. Embrapa - Cerrados. Planaltina - DF. Agosto - 2002

\begin{tabular}{lccccc}
\hline Híbridos & $\begin{array}{c}\text { NFV } \\
\left(\mathrm{n}^{\circ}\right)\end{array}$ & $\begin{array}{c}\text { NFM } \\
\left(\mathrm{n}^{\circ}\right)\end{array}$ & $\begin{array}{c}\text { NFE } \\
\left(\mathrm{n}^{\circ}\right)\end{array}$ & $\begin{array}{c}\text { CF3 } \\
(\mathrm{cm})\end{array}$ & $\begin{array}{c}\text { CPF3 } \\
(\mathrm{cm})\end{array}$ \\
\hline AAG x GOA & $9,05 \mathrm{a}$ & 5,33 & $14,38 \mathrm{a}$ & $250,83 \mathrm{a}$ & $38,00 \mathrm{a}$ \\
AVG x GBRPF & $8,05 \mathrm{a}$ & 4,49 & $12,54 \mathrm{a}$ & $240,00 \mathrm{a}$ & $38,08 \mathrm{a}$ \\
\hline CV(\%) & 17,0 & & 25,7 & 27,0 & 27,0 \\
DMS & 1,49 & & 3,54 & 24,8 & 10,5 \\
(Tukey a 5\%) & & & & \\
Teste F & $8,60 \mathrm{~ns}$ & & $5,00 \mathrm{~ns}$ & $3,89 \mathrm{~ns}$ & $0,0 \mathrm{~ns}$ \\
\hline
\end{tabular}

Médias seguidas de letras diferentes na coluna diferem estatisticamente, pelo teste de Tukey, a $5 \%$ de probabilidade. ns - não significativo. NFM - Não foi feita análise estatística.
Das cultivares testadas, o 'Anão-verde-de-jiquí' vem se destacando no crescimento da planta em altura e circunferência do coleto.

As cultivares anãs apresentam crescimento médio em altura de $5,77 \mathrm{~cm} / \mathrm{mês}$ e $1,45 \mathrm{~cm} /$ mês na circunferência do coleto.

Os híbridos apresentam crescimento médio em altura de 8,88 $\mathrm{cm} / \mathrm{mês}$ e $2,49 \mathrm{~cm} / \mathrm{mês}$ na circunferência do coleto.

\section{REFERÊNCIAS BIBLIOGRÁFICAS}

ARAGÃO, Disponível em: <http:// www.cpatsa.embrapa.br/livrorg/ coco.doc>

JUCÁ, M.P.; GAIIVA, H. N.; PEREIRA, W. E.; MILESKI, A. Comportamento vegetativo de seis cultivares de coqueiro-anão (Cocos nucifera L.), em Santo Antônio de Leverger - MT. Revista Brasileira de Fruticultura, Jaboticabal - SP, v.24, n.2, p.463-467, 2002.

LIMA, M. M. Fruticultura é moda ou uma boa ? Jornal do Produtor, Brasília-DF, n.2, p. 2-3, 1995. Suplemento.

MAHINDAPALA, R.; PINTO, J.L. J. G.Coconut cultivation. Lunuwila: Coconut Research Institute, 1991. 162 p.

MARCÍLIO, H. de C.; GAIVA, H. N.; ABREU, J. G. de.; ARAGÃO, W.M.; FRESCHI, J.C. Avaliação de caracteres vegetativos de híbridos de coqueiro (Cocos nucifera L.) na região não pantanosa do Município de Poconé-MT. Revista Brasileira de Fruticultura, Jaboticabal SP. v.23, n.2, p.437-440, 2001.

PASSOS, E.E.M. Morfologia do coqueiro. In: FERREIRA, J. M. S.; WARWICK, D. R. N.; SIQUEIRA, L. A. A cultura do coqueiro no Brasil. 2. ed. rev. Brasília: Embrapa-SPI; Aracaju: Embrapa-CPATC, 1998a. p. 57-64

PASSOS, E.E.M. Ecofisiologia do coqueiro. In: FERREIRA, J. M. S.; WARWICK, D. R. N.; SIQUEIRA, L. A. A cultura do coqueiro no Brasil. 2. ed. rev. Brasília: Embrapa-SPI; Aracaju: Embrapa-CPATC, 1998b. p. 65-72. 